\title{
3D Digital Technologies for Virtual Fitting of Garments in Tailor-Made Application
}

\author{
Eric R. HARVEY*1, Jocelyn BELLEMARE**2, Denis BOULANGER ${ }^{1}$, \\ Isabelle LESSARD ${ }^{3}$, Philippe PROULX ${ }^{4}$ \\ ${ }^{1}$ CIMMI, Quebec City (QC), Canada; \\ ${ }^{2}$ UQAM, Montreal (QC), Canada; \\ ${ }^{3}$ Vestechpro, Montreal (QC), Canada; \\ ${ }^{4}$ Cégep de Sainte-Foy, Quebec City (QC), Canada \\ http://dx.doi.org/10.15221/14.399
}

\begin{abstract}
Digital 3D technologies are very powerful tools in many fields of application. Apparel industry has been adopting these technologies in order to optimize its design and fabrication processes. Recently, these digital approaches have been applied to try-on stages by using body scanners and are now foreseen for fitting of tailor-made garments.
\end{abstract}

Virtual try-on consists of performing the registration of two 3D models: a human model and a garment model to try-on. Different methods of registration can be used, which have been well described in literature. The precision of the registration depends on the selected method. In the case where the old-fashion tailor-made work is involved, usually the tailor performs manual measurements using a measuring tape directly on the customer. Then a set of measurements is created and the tailor-made garment can be fabricated.

This research explores the use of 3D technologies for performing virtual measurements and try-on. A friendly user 3D manipulation software tool has been developed, which performs virtual measurement using scanned data of the actual body. The set of measurements can be sent to a customer database which includes scanned data and mensuration. A second software tool will be used to perform the try-on for fitting a virtual garment. Different adjustments on the garment are possible by using control points of deformation on the meshed model. The try-on involves the creation of a virtual mannequin based on the customer mensuration.

Keywords: Mass customization, 3D body scan, garment fitting, tailor-made, virtual try-on

\section{Introduction}

Consumers are better educated and more demanding nowadays. They are no longer satisfied with standardized products that force them to compromise. The Internet influences customers' buying habits by creating needs that have to be satisfied instantaneously. In the clothing industry, these expectations not only imply having to constantly provide consumers with new options in terms of styles and colors, but also to allow them to find an affordable close-fitting clothing item and make it available to them almost as rapidly as if it was a standard-sized product. In order to meet these expectations, clothing companies must now propose custom-made products. Brands that offer personalized products (mass customization) are taking over both conventional and online stores. This is made possible by identifying measurement key points necessary to produce well adjusted, close-fitting garments. However, being able to take measurements efficiently and effectively is crucial.

To meet customer expectations, clothing companies now have to manufacture or offer tailor-made products. Brands that offer mass customization products are spreading through traditional businesses as well as online. In the clothing industry, some companies have successfully applied mass customization principles to their formerly standardized products.

\footnotetext{
* eharvey@cimmi.qc.ca; +1-418-781-2464; www.cimmi.qc.ca

** bellemare.jocelyn@uqam.ca; +1-514-933-6988; www.uqam.ca
} 
Although efficient and affordable technologies are available to provide a body scan, few businesses are able to meet the requirements of custom-made products for the following reasons:

- lack of reliability of the measurements provided by the body scanner;

- bandwidth related issues due to the transmission of large quantity of data to potential manufacturers;

- issues related to interfacing the data required by the sewing pattern making and the raw data obtained by the body scanner;

- issues related to scale and aspect mismatches between the human model template and the actual customer body.

In the apparel industry, some companies have successfully resolved issues with mass customization principles to products that were thus far standardized. Even manufacturers and designers such as tailors have played the role of pioneers in the luxury goods market by offering prêt-à-porter and customizable tailor suits.

Apparel industry has been adopting 3D technologies, which are very powerful tools in many fields of applications, in order to optimize the design and fabrication processes. Recently, these technologies have been applied to try-on stage by using body scanners and are now foreseen for tailor-made fitting of garments. Nevertheless, mass customization somehow remains misunderstood or is rarely used by actors in the clothing industry mainly because of issues described previously and of the need for flexibility of manufacturing delays and methods.

The objectives of the present study are to help in the manufacturing of tailor-made garments by using $3 \mathrm{D}$ technologies for performing virtual measurement and try-on. These technologies are intended to be used to analyze measurement data coming from peripherals such as a body scanner in order to identify as precisely as possible the necessary information to produce a well-fitting garment. They are also intended to perform try-on tests for fitting a virtual garment over a virtual precise model of the subject body. Different adjustments on the garment are possible by using deformation control points on its virtual counterpart.

In this context, the specific objective of this project is to identify the data that are necessary to produce a satisfying virtual tailor-made garment with fundamental variables such as length, circumference, density and textile matter behavior that will allow to significantly diminishing the amount of data to analyze and to send out in order to create a smart pattern. Moreover, using anthropometric measurements, like shape and density, will enable us to identify key referential points which are essential to ensure proper fit. These points, when combined with data related to textile textures and behaviors, will allow personalized pattern grading.

Ultimately, this project aims at reducing the amount of data while increasing the quality of digital 3D technologies like automated garment patterns, thereby allowing the production of well-adjusted tailor-made garments that meet customers' expectations.

\section{Mass customization using digital technologies}

Both methods of body measurement, manually or automated, have their strengths and weaknesses. For some authors the biggest strength of the manual approach is its ability to identify incoherent measurements while its most important weaknesses are the labour costs and the imprecision caused by human error when transcribing data [1]. On the other hand, the strengths of the automated approach are the speed and the low cost (nowadays) while its main weakness is in the measurement inconsistencies due to movement [5], the lack of accuracy when compared with manually measurements [12], and the difficulty to obtain correct measurements at feet level for instance [9]. Accurate body measurements can be difficult to obtain with 3D body scanning due to factors such as posture, landmark indications, instruments position and orientation, pressure and tension exerted [1].

Not so long ago, body scanning was still at the stage of acceptance and maturation in the industry. The benefits of automation were not clearly visible [9]. Female consumers who have been scanned generally react well to the results, yet women from specific socio-demographic groups are less comfortable with the idea of being body scanned [8].

As Whitestone \& Robinette note [15], 3D body scanning is now an accepted tool and as time goes by, the non-contact body measuring technologies generate more and more interest and applications in the apparel industry. It can be utilized for numerous applications: anthropometric measuring surveys, 
development of three-dimensional apparel, computer aided design (CAD), virtual garment environments and animation, mass-customization, etc. [6,3,13,7,16,14].

Guan and al. [2] propose a framework shown in Figure 1, which represents a virtual clothing shop which meets the mass customization based on virtual 3D clothing design model. To incorporate the respective merits both online and custom-tailoring shops, the framework will be used to establish a new interactive model which consists of virtual human, virtual clothing and virtual interaction.

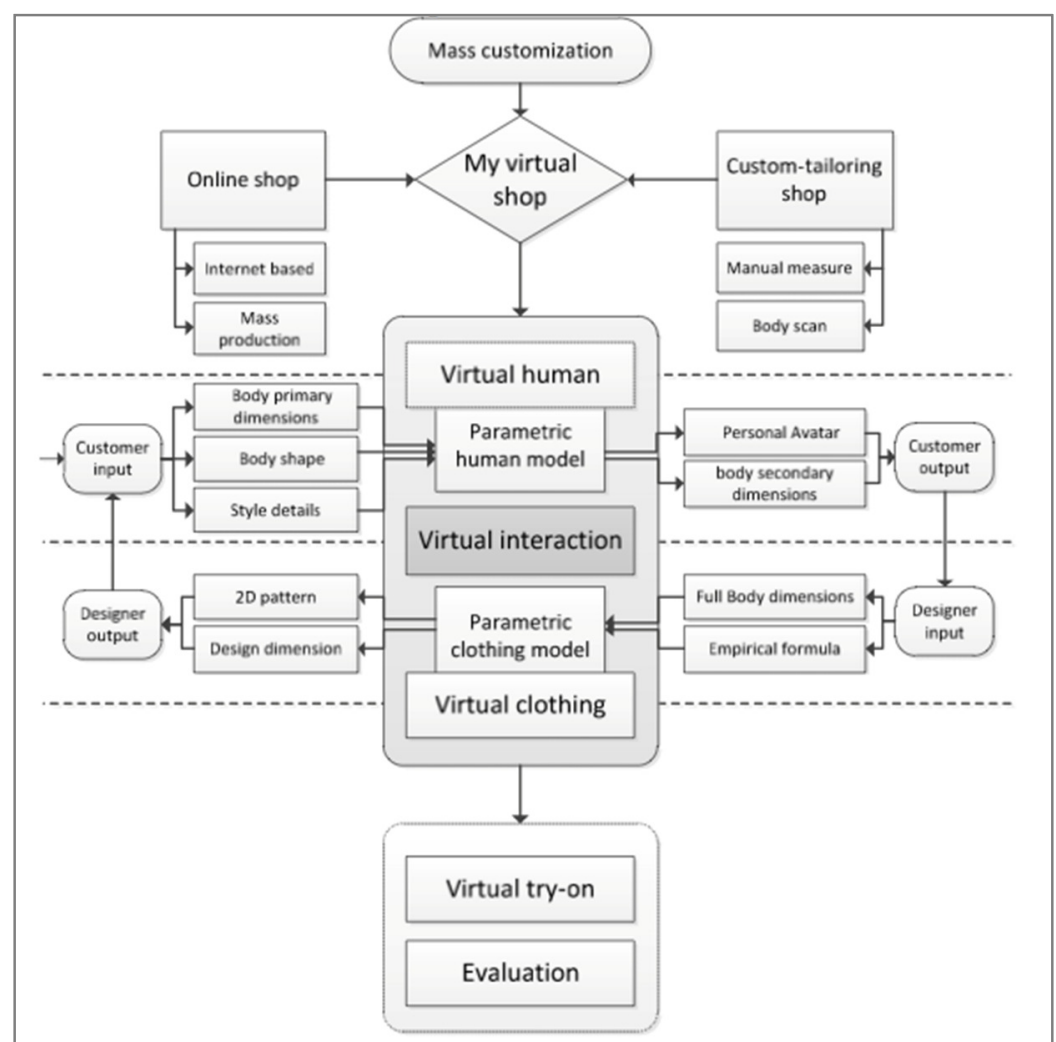

Fig. 1 The framework of the virtual shop (Guan \& al., 2012).

\section{Research on digital tools with 3D technologies}

Within the framework of a research grant from the Ministry of Enseignement Supérieur, de la Recherche et de la Science du Québec (MESRS) and with the support of an industrial partner, our research explores the use of $3 \mathrm{D}$ technologies for performing virtual measurement and try-on. A friendly user 3D manipulation software tool has been developed, which performs virtual measurement using scanned data of the actual body. The set of measurements can be sent to a customer database which includes scanned data and mensuration. A second software tool will be used to perform the try-on for fitting a virtual garment. Different adjustments on the garment will be possible by using control points of deformation on the meshed model. The try-on will involve the creation of a virtual mannequin based on the customer mensuration.

\subsection{Methodology}

Firstly, the approach consisted of determining the process requirements of our industrial partner involved in the tailor-made market. This allowed defining specific requirements in terms of virtual tools, which would be needed to accomplish measurement and try-on tasks together with the manual way consisting of using a measuring tape.

Secondly, a software platform with simple graphical user interface has been developed for importing and manipulating 3D scan data from body scanners. The scan data issued from a body scanner usually consist of a 3D point cloud, which is meshed to form a polygonal model in OBJ format. Starting with a basic data manipulation tool, functions for simulating a measurement tape have been added to the software platform. The functions allow navigating through the 3D data, positioning a cross section at specific measurement region of the body and selecting control points for adjusting the angular orientation of the virtual tape. 
Thirdly, a database of body scan data will be used for comparison between actual tape measurements performed on real person and these measurements virtual counterparts using the measurement software tool. The error between the two types of measurement will be evaluated in terms of scanner accuracy and positioning of the measuring tape between the real human and its virtual counterpart.

\subsection{Process of virtual measurement and try-on}

The conventional tailoring method enables the tailor to take professional measurements and to assess posture and body shape for making unique modifications to the garment. Local tailors will typically have a showroom allowing customers to choose fabrics from samples or return the garment easily should it require further modification.

Tailoring has evolved to e-commerce, which involves ordering a garment from an out-of-town tailor enabling cheaper labor to be used. In practice this can now be done on a global scale via websites. Unlike local tailoring, customers must take their own measurements, fabric selection must be made from a photo and if further alterations are required the garment must be shipped.

A further step of tailoring evolution will consist of customer's virtual measurement and try-on. As shown in Figures 2 and 3, the processes aim at gathering 3D models and measurements which can be stored in a customer's personalized database. Then eventually the next step in the process will consist of producing and sending digital pattern pieces to a local or a remote garment manufacturer.

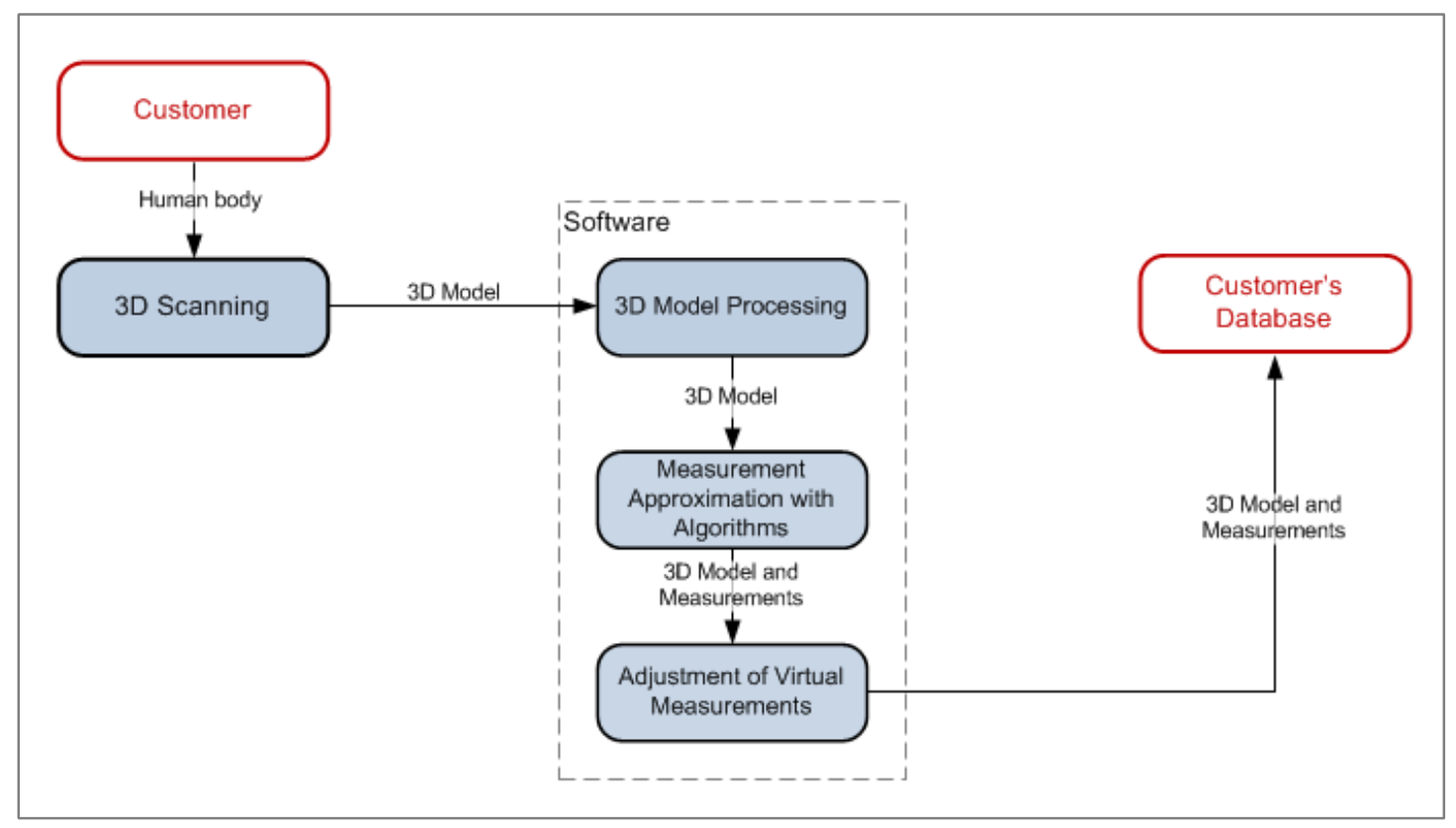

Fig.2 Virtual measurement process.

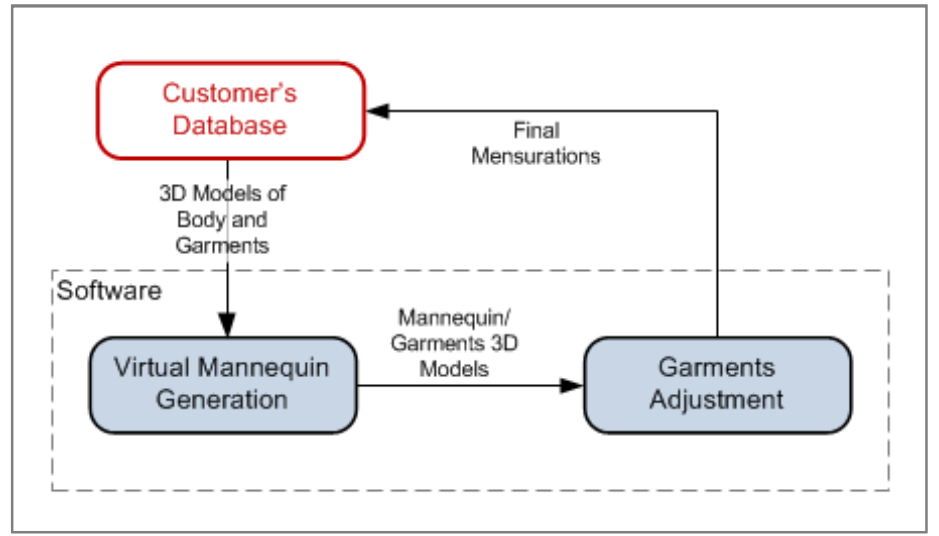

Fig.3 Virtual try-on process. 


\subsection{Digital tools development for tailor-made application}

Configuration is an essential aspect of mass customization because it creates the possibilities to guide customers as they are making choices. Piller [11] contends that the primary objective of a configurator is to ease the decision-making process of customers using a Web-based interface. Product configuration systems play an important role in supporting the mass customization paradigm, as it helps to determine the degree of personalization that a business will offer. Thus, the role of the virtual measurement and try-on tools is to create a link between consumers and manufacturers. For Inala [4], mass customization does not equate to an increase in costs. According to Moser and Piller [10], using a configurator could significantly reduce costs since its Web-based technology diminishes the time required to take orders.

Figures 4-7 demonstrate how the virtual tools would operate in clothing customization process. One of the software tools called measurement tool (Figures 4 and 5) is used for importing a scan data set converted into a meshed model and for performing measurements at selected cross sections along the body. A user-friendly interface has been developed for providing very simple body measurement steps.

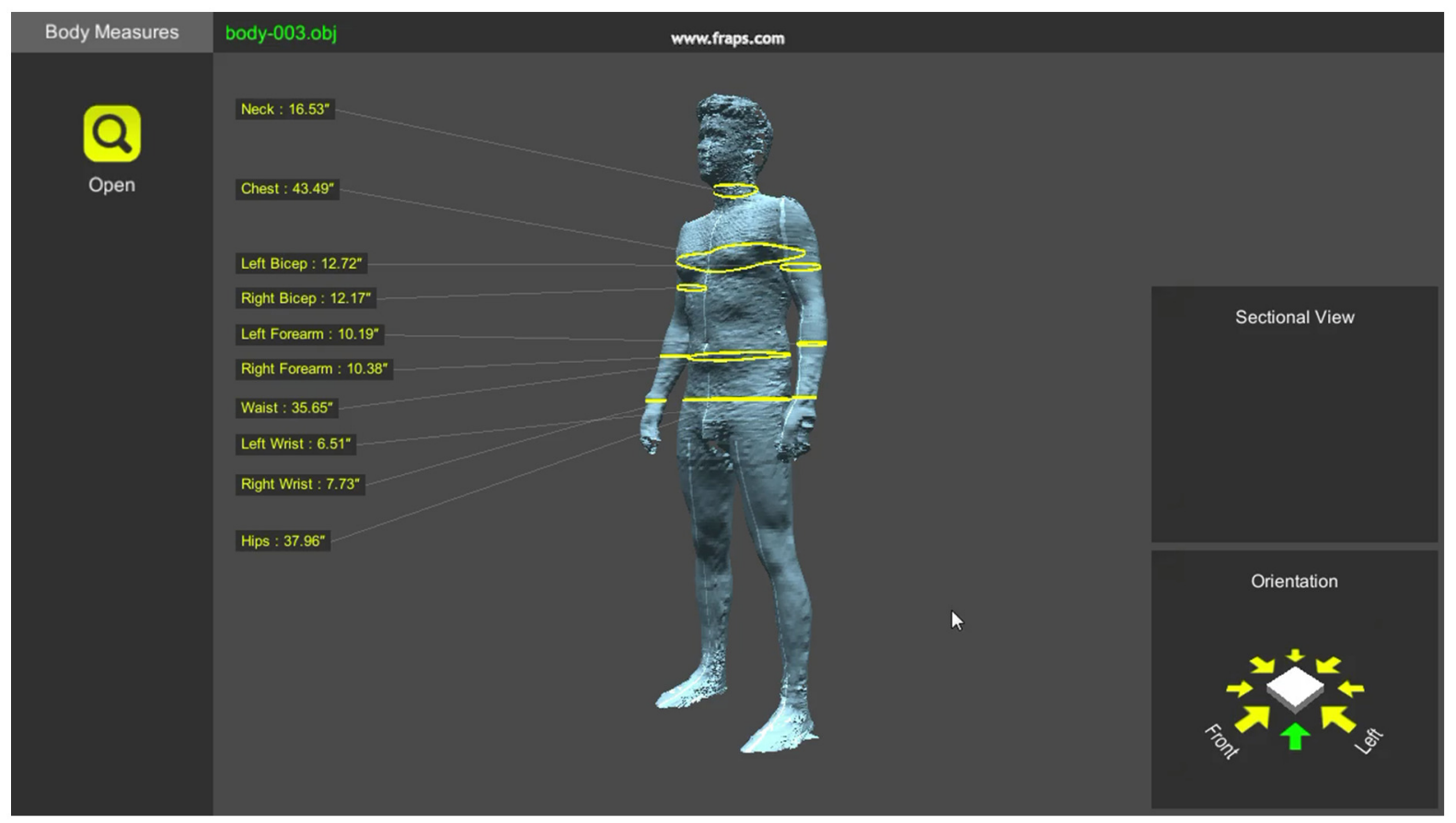

Fig. 4. Software tool for body measurements.

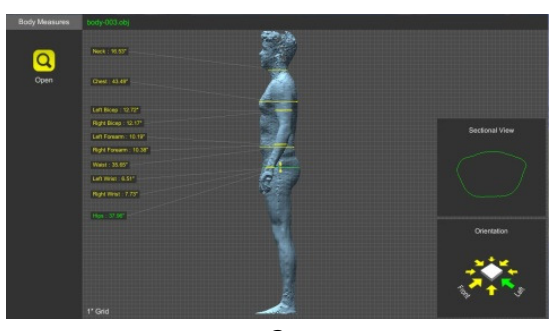

a.

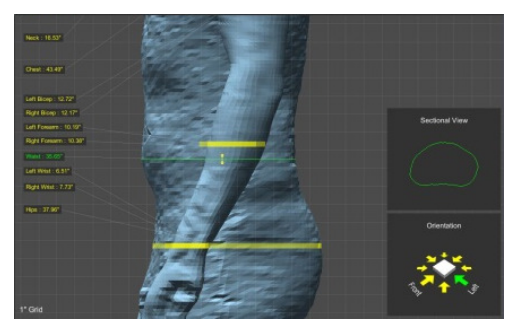

b.

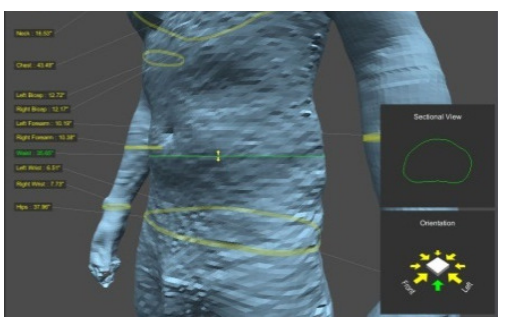

C.

Fig. 5. Body measurements steps: a.Opening of the 3D model file; $b$. Clicking of the measurement zone; $c$. Adjustment of the virtual measuring tape position with control points (green line with small arrows).

A second digital tool has been simulated using a 3D modeling software (Autodesk 3DS Max) for showing the possibilities of try-on for fitting a virtual garment. Figures 6 and 7 present what is envisioned for performing virtual try-on. This particuliar example involving a digital shirt shows how different adjustments on the garment are possible by using deformation control points on the meshed model. 


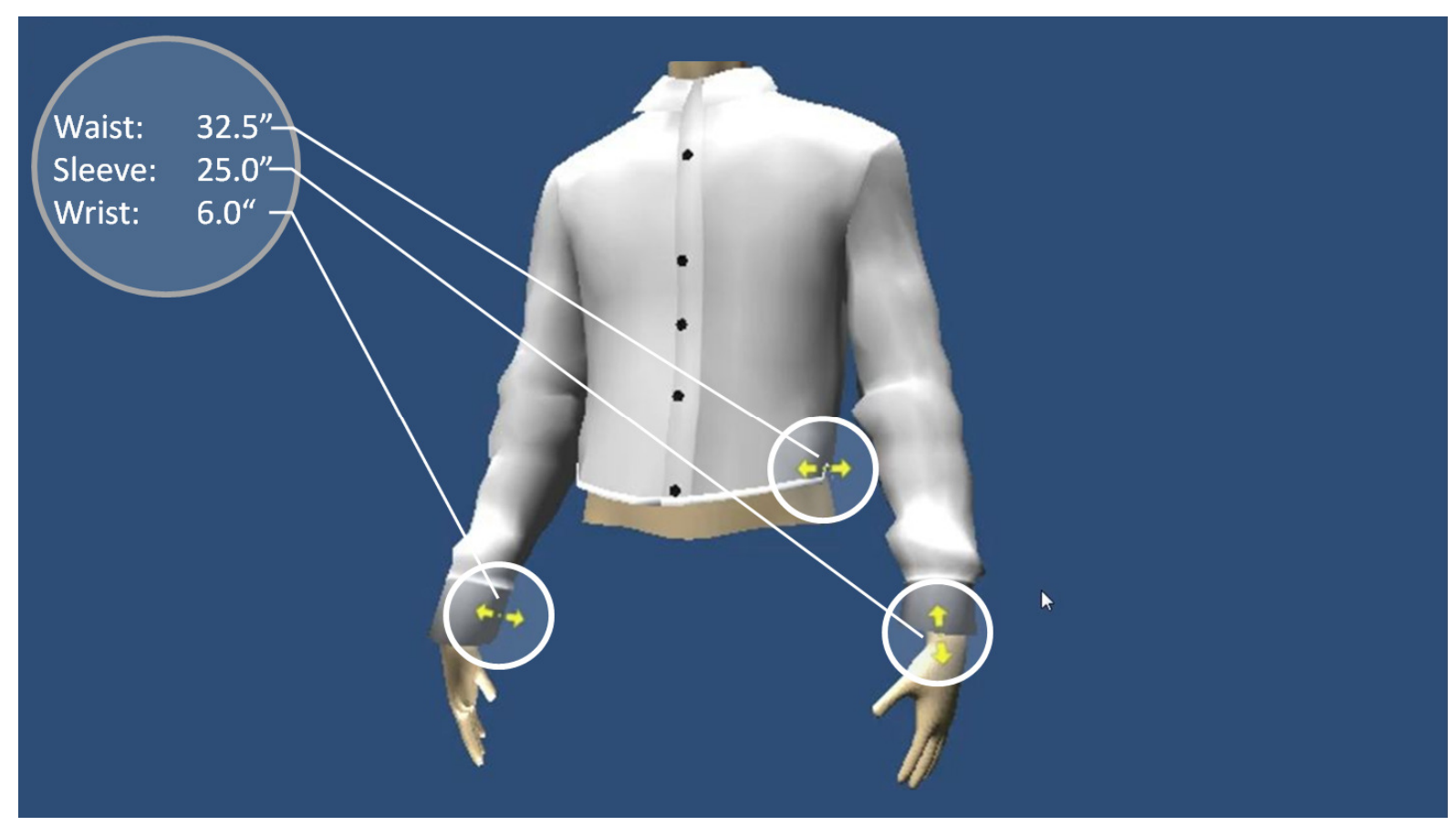

Fig. 6. Simulation of virtual try-on.
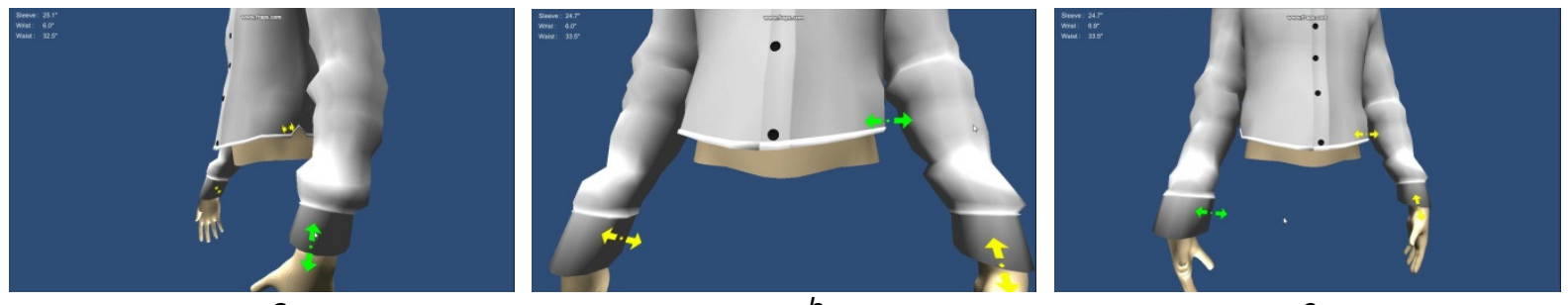

Fig. 7. Body measurements steps: a.Opening of the 3D model file; $b$. Selection of the adjustment zone; c. Virtual adjustment of the wrist with control points (green arrows).

The virtual measurement and try-on tools must be used alongside with a high-performance technological platform to allow interaction between the customer, the tailor and the garment manufacturer as the product is designed. This interface link creates opportunities for value co-creation in both the apparel and fashion industries.

\section{Conclusion}

This research project offers great possibilities in terms of innovation and could constitute an outstanding opportunity for several actors in the clothing industry. If prime producers want to make the most of this prospect, they would need a better understanding of what can be done in terms of clothing personalization and mass customization, so as to formulate an appropriate strategy on how to use their 3D technologies for performing virtual measurement and try-on. From the start, mass customization needs to directly involve customers in the designing and manufacturing phases. Moreover, this customization model must provide opportunities to generate savings by reducing stocks and by allowing a better integration of all actors in the supply chain. Mass customization with 3D technologies offers possibilities to reach, or even surpass, customers' expectations.

This research project allowed to understand the needs of the tailoring industry and to contribute to the development of digital tools which perform virtual measurements on a scanned body. The next step will consist of developing a software tool for the try-on involving digital garments and a virtual mannequin. This will provide tools for the apparel businesses allowing them to gain a competitive edge through custom-made garments. The opportunities created by the absence of such a service should be used by these businesses to reposition themselves on the garment and apparel markets, both locally and internationally. 


\section{Acknowledgements}

We would like to acknowledge the financial support from the Ministry of Enseignement Supérieur, de la Recherche et de la Science du gouvernement du Québec (MESRS).

\section{References}

[1] Fan, W.J. \& Hunter, L., Clothing Appearance and Fit: Science and Technology, Woodland, Publishing Ltd., Cambridge, 2004.

[2] Guan, C., Xu, B., Qin, S. \& Wang, S., A new apparel design and online shopping framework for mass customization and best fit, Proceedings of the $18^{\text {th }}$ international conference on automation \& computing, Loughborough university, Leicestershire, UK, pp. 1-5, 2012.

[3] Hardaker, C.H.M. \& Fozzard, G.J.W., Towards the virtual garment: three-dimensional computer environments for garment design, International Journal of Clothing Science and Technology, 10 (2): 114-12, 1998.

[4] Inala, K., Assessing product configurator capabilities for successful mass customization, University of Kentucky, theses, 2007.

[5] Istook, C. L., \& Huang, S., 3D body scanning systems with application to the apparel industry, Journal of Fashion Marketing and Management, 5 (2): 120- 132, 2001.

[6] Jones, P. R. M., Li, P., Brooke-Wavell, K., \& West, G., Format for human body modeling from 3-D body scanning, International Journal of Clothing Science and Technology, 7 (1): 7-16, 1995.

[7] Koontz, M.L., \& Gibson, I.E., Mixed Reality Merchandising: Bricks, Clicks and-Mix, Journal of Fashion Marketing \& Management, 6 (4): 381-395, 2002.

[8] Loker, S., Ashdown, S. P., Cowie, L., \& Schoenfelder, K. A., Consumer interest in commercial applications of body scan data, Journal of Textile and Apparel, Technology and Management, 4 (1): 1-13, 2004.

[9] McKinnon, L. \& Istook, C., Body Scanning: the effects of subject respiration and foot positioning on the data integrity of scanned measurements, Journal of Fashion Marketing and Management, 6 (2): 103-121, 2002.

[10]Moser, K., Piller, F.T., The international mass customization case collection: an opportunity for learning from previous experiences, Journal: Int. J. of Mass Customization, 2006 Vol.1, No.4, pp. 403 - 409, 2006.

[11]Piller, F., Mass customization: reflections on the state of the concept, International Journal of Flexible Manufacturing Systems, 16 (4): 313-34, 2004.

[12] Simmons, K.P., Istook, C.L., Body measurement techniques: Comparing 3D body-scanning and anthropometric methods for apparel applications, Journal of Fashion Marketing and Management, Vol. 7 Iss: 3, pp.306 - 332, 2003.

[13] Stylios, G.K., Han, F., \& Wan, T.R., A remote, on-line 3-D human measurement and reconstruction approach for virtual wearer trials in global retailing, International Journal of Clothing Science and Technology, 13 (1): 65-75, 2001.

[14]Ulrich, P. V., Anderson-Connell, L. J. \& Wu, W., Consumer co-design of apparel for mass customization, Journal of Fashion Marketing \& Management, 7 (4): 398-412, 2003.

[15]Whitestone, J. J., \& Robinette, K. M., Fitting to maximize performance of HMD systems, In J. Melzer \& K. Moffit (Eds.), Head-Mounted Displays: Designing for the User, New York: McGraw-Hill, 206-215, 1997.

[16]Xu, B., Huang, Y., Yu, W., \& Chen, T., Three-dimensional body scanning system for apparel masscustomization, Opt. Eng., 41 (7): 1475-1479, 2002. 